\title{
A candidate probiotic with unfavourable effects in subjects with irritable bowel syndrome: a randomised controlled trial
}

\author{
Solveig C Ligaarden ${ }^{1,2^{*}}$, Lars Axelsson ${ }^{3}$, Kristine Naterstad ${ }^{3}$, Stian Lydersen², Per G Farup ${ }^{1,2}$
}

\begin{abstract}
Background: Some probiotics have shown efficacy for patients with irritable bowel syndrome (IBS). Lactobacillus (L.) plantarum MF1298 was found to have the best in vitro probiotic properties of 22 strains of lactobacilli. The aim of this study was to investigate the symptomatic effect of L. plantarum MF1298 in subjects with IBS. Primary outcome was treatment preference and secondary outcomes were number of weeks with satisfactory relief of symptoms and IBS sum score.

Methods: The design was a randomised double blind placebo-controlled crossover trial. 16 subjects with IBS underwent two three-week periods of daily intake of one capsule of $10^{10}$ CFU L. plantarum MF 1298 or placebo separated by a four-week washout period.

Results: Thirteen participants (81\%; 95\% Cl 57\% to 93\%; $P=0.012$ ) preferred placebo to L. plantarum MF1298 treatment. The mean (SD) number of weeks with satisfactory relief of symptoms in the periods with L. plantarum MF1298 and placebo were $0.50(0.89)$ and $1.44(1.26)$, respectively $(P=0.006)$. IBS sum score was $6.44(1.81)$ in the period with L. plantarum MF1298 treatment compared with $5.35(1.77)$ in the period with placebo $(P=0.010)$. With a clinically significant difference in the IBS sum score of 2 in disfavour of active treatment, the number needed to harm was $3.7,95 \% \mathrm{Cl} 2.3$ to 10.9 .
\end{abstract}

Conclusions: This trial shows for the first time an unfavourable effect on symptoms in subjects with IBS after intake of a potential probiotic.

The trial registration number: Clinical trials NCT00355810.

\section{Background}

Irritable bowel syndrome (IBS) is the most frequent functional gastrointestinal disorder, with a prevalence of $5-11 \%$ in most countries [1]. The workload generated by IBS is considerable and constitutes approximately onethird of all visits to gastroenterologists [2]. It is a biopsychosocial disorder that requires a multifactorial approach [3]. No proper treatment is available.

The human gut contains over 1000 different bacterial species and an indeterminate number of strains of which a minority of the strains is cultivable [4] Probiotics are defined as "live microorganisms which when administered in adequate amounts confer a health benefit on the host" [5]. Some studies have shown beneficial

\footnotetext{
* Correspondence: solveig.ligaarden@ntnu.no

'Department of Medicine, Innlandet Hospital Trust, Gjøvik, Norway
}

effect of probiotics in IBS [6]. Lactobacillus (L.) plantarum 299v reduced flatulence and abdominal pain in patients with IBS [7]. L. plantarum MF1298 was found to have the best in vitro probiotic properties of 22 strains of lactobacilli isolated from fermented food products. This strain was confirmed to adhere to the human colon adenoma cell line $\mathrm{CaCo} 2$, to strengthen transepithelial resistance of a $\mathrm{CaCo} 2$ cell layer and to increase production of certain tight junction proteins, to have antimicrobial activity against potential pathogens, and to survive passage through the human gastrointestinal tract [8-10]. L. plantarum MF1298 was therefore proposed as a potential candidate probiotic strain.

The aims of this randomised placebo-controlled crossover trial were to study the effect of L. plantarum 
MF1298 on treatment preference, satisfactory relief of symptoms and symptoms in subjects with IBS.

\section{Methods \\ Participants}

Participants were recruited from a hospital-based gastroenterology outpatient clinic and a private gastroenterological practice. Subjects 18 to 75 years of age with IBS according to the Roma II criteria and symptoms the last three months were eligible for inclusion. All subjects had had a sigmoidoscopy or colonoscopy performed within the last five years to exclude organic disease. Other tests to confirm the diagnosis were performed at the physicians' discretion. Pregnant and breast-feeding women and subjects with major psychiatric, mental or behavioural disorders, coexisting gastrointestinal and other disorders that might influence the symptoms, or poor knowledge of language were excluded, as were those who had used probiotics more than once a week in the previous three weeks or antibiotics or laxatives in the previous five weeks. The study was made in accordance with the Helsinki Declaration and all participants gave written informed consent to participation before enrolment. The Regional medical research ethics committee, Central Norway approved the study protocol.

\section{Study design}

The study was a randomised double blind, placebo-controlled, crossover trial with a one-week run-in period followed by randomisation and two three-week treatment periods separated by a four-week washout period. Participants with satisfactory relief of symptoms in the run-in period were excluded from further participation. IBS symptoms were recorded on diary cards every evening during the run-in period, during the last week of the washout period, and the last week of the two treatment periods. Satisfactory relief of symptoms was recorded on diary cards at the end of the run-in and washout periods and at the end of each week during the treatment periods. At the end of the study, the participants recorded treatment preference for one of the treatment periods. Faecal samples were collected at the end of the run-in, washout period, and the two treatment periods. All data were collected at the hospital based gastroenterology outpatient clinic at Innlandet Hospital Trust, Gjøvik.

The computer-based randomisation was performed at the Unit for Applied Clinical Research, Norwegian University of Science and Technology, Trondheim, Norway. Faun Pharma, Norway, provided packed and numbered containers with the capsules containing $10^{10} \mathrm{CFU}$ live, freezedried L. plantarum MF 1298 or placebo according to the randomisation list. The capsules were confirmed to contain the correct number of pure L. plantarum MF1298 by classical and genetic methods, and were checked for the presence of common pathogens. The capsules looked identical and were prescribed to be taken once daily with liquid. The participants and health care providers were blinded until data entry was complete.

\section{Assessments}

The participants were asked about treatment preference (the period with least symptoms) at the last visit, and about satisfactory relief of symptoms (yes/no) at the end of the run-in and washout periods and at the end of each week during the treatment periods. Seven gastrointestinal symptoms were recorded. Abdominal pain/discomfort, urgency and bloating were recorded as none, mild, moderate, or severe (score 0-3); stool frequency as number of stools per day; stool consistency according to Bristol stool scale form (score 1-7); and straining and incomplete bowel movement as yes/no (score: 1 or 0 ) [11]. An IBS sum score (score 0-15) was calculated as the sum of these seven scores after "normalisation" of stool frequency and consistency to achieve low scores for normal bowel habits. The "normalisation" was performed as follows: Stool frequency: 0 stool $/$ day $=1 ; 1-3$ stools $/$ day $=0$; 4-5 stools $/$ day $=1 ; \geq 6$ stools $/$ day $=2$. Stool consistency: Bristol stool scale 3-5 $=0$; Bristol stool scale 2 and $6=1$; Bristol stool scale 1 and $7=2$. A diarrhoea score was calculated as the sum of the "none normalised" scores of stool frequency and stool consistency. Assessment of compliance was based on returned capsules.

Faecal samples, frozen in Carey Blair medium (Oxoid Ltd, Basingstoke, Hampshire, UK), were analyzed for detection of $L$. plantarum by real-time PCR using 50 cycles. Primers: 5'-TGG ACC GCA TGG TCC GAG-3' (F) and 5'-GTG AGC CGT TAC CCC ACC AT-3' (R), and the Taqman probe 5'-TCC CGC GGC GTA TTA3 , targeting a specific $L$. plantarum region of the conserved 16S rDNA sequence, were used in the analysis. Verification of specificity and control of efficiency of the primer-probe pair were performed according to standard procedures, and will be documented elsewhere (Rudi et al., manuscript in preparation).

\section{Outcomes}

The primary outcome measure was treatment preference. Secondary outcomes were the number of weeks with satisfactory relief of symptoms and the IBS sum score. All comparisons were between treatment with $L$. plantarum MF1298 and placebo. Presence of L. plantarum MF1298 was assessed by analysis of faeces as described above. Adverse events were noted.

\section{Statistical methods}

The sample size calculation was based on the treatment preference (the proportion of participants preferring one 
treatment period to the other). Nineteen participants were required to reveal a treatment preference of $80 \%$ compared with the null hypothesis value of $50 \%$, with $80 \%$ power at a two-sided significance level of $5 \%$.

Changes within and between groups were compared with paired $t$ test and independent $t$ test, respectively. The confidence interval and $P$ value for treatment preference were calculated with the Wilson (score) method. The difference between treatment periods as regards number of subjects with satisfactory relief of symptoms for zero, one, two and three weeks was tested with the marginal homogeneity test for matched ordinal variables. The number needed to harm was calculated by the method described by Walter, with a difference $\geq 2$ in the IBS sum score between L. plantarum MF1298 and placebo regarded as clinically significant [12]. Twosided $P$ values $<0.05$ were considered statistically significant, and the $95 \%$ confidence interval (CI) was calculated for the main outcomes. Modified intention-to-treat analysis was performed. All results are given as mean (SD) unless otherwise indicated.

\section{Results}

Twenty-eight participants were included between January and April 2006. Figure 1 shows the flow of participants through the trial. Sixteen participants (five males and eleven females) with a mean age of 50 (11) years and BMI 24 (3) $\mathrm{kg} / \mathrm{m}^{2}$ were available for the modified intension-to-treat analysis; one had constipation-predominant, nine alternating, and six diarrhoea-predominant IBS. The IBS sum score at run-in was 6.21 (1.63), and the duration of symptoms was 31 (17) years. Four participants with protocol violations were included in the modified intention-to-treat analyses; two had inadequately completed diary cards (three and five days, respectively), one used an antibiotic during the active period, and one received supplementary treatment for IBS in the placebo period.

Thirteen participants $(81 \%$; CI $57 \%$ to $93 \% ; P=0.012$ ) preferred placebo to L. plantarum MF1298 treatment. The number of weeks with satisfactory relief of symptoms was statistically significantly higher in the placebo period compared with the L. plantarum MF1298 period (Table 1 and 2) and the IBS sum score and the score for diarrhoea were significantly higher in the period of L. plantarum MF1298 treatment than with placebo (Table1). The subclasses of IBS (diarrhoea predominant, constipation predominant, and alternating) showed the same tendency for higher IBS sum scores in the period with L. plantarum MF1298 compared with placebo (data not shown). Figure 2 shows the IBS sum scores during the trial by allocation group. The IBS sum score in the active period was 6.44 (1.81), in the placebo period 5.35 (1.77), and the correlation between these was 0.66 . The difference in IBS sum score between active treatment and placebo was 1.09 (1.47). The resulting proportion of subjects with a score difference of at least 2 in disfavour of active treatment was $27 \%$ (CI $9 \%$ to $44 \%$ ), and the number needed to harm was 3.7 (CI 2.3 to 10.9 ).

L. plantarum was not detected in the faeces in any of the subjects in the run-in period, in the washout period (except for in one subject given active treatment in the first period), nor in the placebo period. However, L. plantarum was detected in all faecal samples at the end of the active treatment period, indicating that the analysis was targeting strain MF1298.

Compliance with intake of drugs was $95 \%$. Two participants did not return their unused drugs after the last treatment period.

One participant had a short stay in hospital for cervicobrachialgia during the washout period, two weeks after the end of active treatment. There was no organic explanation and she continued in the trial. Three minor adverse events were noted.

\section{Discussion}

The study shows an unfavourable effect on symptoms in subjects with IBS after intake of $L$. plantarum MF1298 compared to placebo. To our knowledge, similar unfavourable effects of probiotics have not been reported in subjects with IBS. Other studies with probiotics in subjects with IBS show either no effect or a favourable effect [6]. The divergent results could be related to different probiotic properties and health effects of the genera, strains, and species in use. Quigley assumed the possible superiority of Bifidobacterium spp for treatment in IBS [13]. Bifidobacterium (B.) animalis DN-173010 increased stool frequency in subjects with constipation at entry in a large study [14]. O'Mahony et al. compared the symptomatic effect of $L$. salivarius UCC4331 and $B$. infantis 35624 in subjects with IBS [15]. B. infantis 35624 induced a favourable effect on IBS symptoms. In a second study by the same researchers, the beneficial effect of $B$. infantis 35624 was confirmed [16]. Lactobacilli have been evaluated in several trials with inconsistent results, but no deleterious effects have been reported. One trial of $L$. reuteri ATCC 55730 showed no significant effect on gastrointestinal symptoms in patients with IBS, while another trial also in patients with IBS showed no effect of $L$. casei strain GG [17]. $L$. casei strain GG in combination with other probiotics showed a positive effect on IBS symptoms in one study by Kajander [18]. Two trials with L. plantarum 299v showed a reduction of abdominal pain and flatulence, while a small crossover study found no effect on symptoms of IBS [19].

Reports of unfavourable effects of probiotics are rare and probiotics have until recently been regarded as safe 


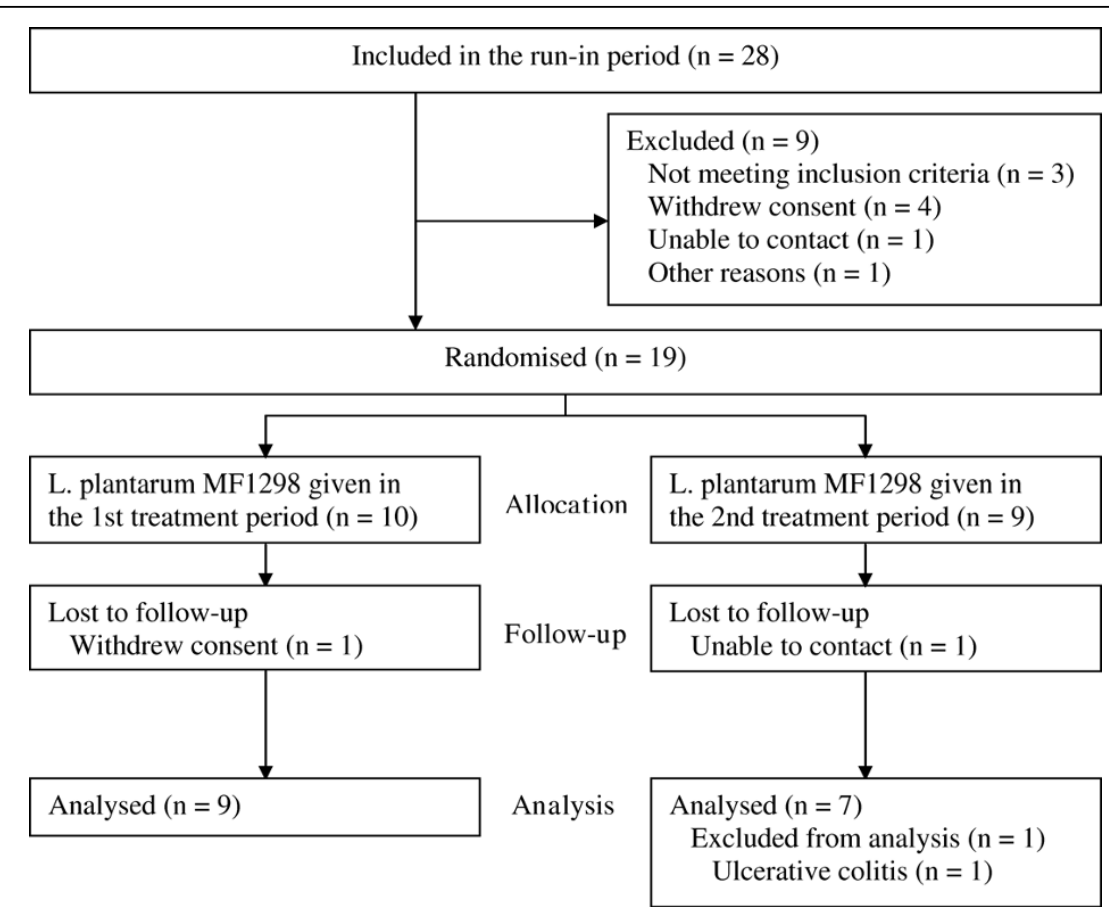

Figure 1 Flow chart of the participants through the trial.

[6]. Untoward effects were reported in only three out of 185 human studies [20]. A strain of L. acidophilus increased faecal protein catabolites in healthy volunteers in one study, while Saccharomyces cerevisiae increased disease activity in patients with stable Crohn's disease in one study, and in another study increased serum glucose in healthy volunteers [20]. Sepsis has been reported in some subjects using probiotics [21]. The most alarming report was published in 2008, showing increased mortality of severe acute pancreatitis following treatment with a multispecies probiotic preparation [22].

The doses of probiotics used for the treatment of IBS in other trials vary from $2 \times 10^{8}$ to $2 \times 10^{10} \mathrm{CFU}$ per day $[7,15,19,23,24]$. In a dose-finding study, the optimal dose of $B$. infantis 35624 was $1 \times 10^{8} \mathrm{CFU}$ which was superior to placebo, $1 \times 10^{6} \mathrm{CFU}$ and $1 \times 10^{10} \mathrm{CFU}$.

Table 1 Daily symptom scores and number of weeks with satisfactory relief of symptoms during the two treatment periods.

\begin{tabular}{|c|c|c|c|c|}
\hline Symptoms & LpMF1298 & Placebo & Paired differences, mean $(\mathrm{Cl})$ & Statistics \\
\hline Number of weeks with satisfactory relief of symptoms & $0.50(0.89)$ & $1.44(1.26)$ & $-0.94(-1.57$ to -0.31$)$ & $P=0.006$ \\
\hline \multicolumn{5}{|l|}{ Individual symptoms } \\
\hline Abdominal Pain/Discomfort & $1.55(0.57)$ & $1.14(0.55)$ & 0.41 (0.09 to 0.73$)$ & $P=0.016$ \\
\hline Stool frequency (normalised) & $0.15(0.18)$ & $0.19(0.21)$ & $-0.03(-0.14$ to 0.07$)$ & $P=0.48$ \\
\hline Stool consistency (normalised) & $0.86(0.55)$ & $0.61(0.55)$ & $0.25(-0.12$ to 0.61$)$ & $P=0.17$ \\
\hline Urgency & $1.54(0.59)$ & $1.12(0.56)$ & $0.42(0.17$ to 0.66$)$ & $P=0.002$ \\
\hline Bloating & $1.23(0.59)$ & $1.16(0.68)$ & $0.07(-0.31$ to 0.46$)$ & $P=0.69$ \\
\hline Straining & $0.51(0.37)$ & $0.58(0.40)$ & $-0.07(-0.17$ to 0.03$)$ & $P=0.13$ \\
\hline Incomplete bowel movement & $0.59(0.35)$ & $0.54(0.41)$ & $0.05(-0.06$ to 0.17$)$ & $P=0.35$ \\
\hline \multicolumn{5}{|l|}{ Sum symptoms } \\
\hline IBS sum score & $6.44(1.81)$ & $5.35(1.77)$ & 1.09 (0.31 to 1.87$)$ & $P=0.010$ \\
\hline \multicolumn{5}{|l|}{ Stool characteristics } \\
\hline Stool frequency & $1.52(0.68)$ & $1.33(0.58)$ & $0.19(-0.07$ to 0.45$)$ & $P=0.15$ \\
\hline Stool consistency & $4.84(1.51)$ & $4.17(1.31)$ & 0.67 (0.20 to 1.13$)$ & $P=0.008$ \\
\hline Diarrhoea (consistency + frequency) & $6.36(1.99)$ & $5.50(1.71)$ & $0.86(0.33$ to 1.39$)$ & $P=0.004$ \\
\hline
\end{tabular}

The results are given as mean(SD). 
Table 2 Number of subjects with satisfactory relief of symptoms for $0,1,2$, and 3 weeks in the two treatment periods. Number of subjects with satisfactory relief of symptoms for $0,1,2$, and 3 weeks Total no. of subjects in the placebo period

\begin{tabular}{|c|c|c|c|c|c|c|}
\hline & & 0 week & 1 week & 2 weeks & 3 weeks & \\
\hline Number of subjects & 0 week & 4 & 4 & 1 & 2 & 11 \\
\hline with satisfactory relief & 1 week & 1 & 0 & 0 & 2 & 3 \\
\hline $\begin{array}{l}\text { of symptoms for } 0,1,2 \text {, and } \\
3 \text { weeks }\end{array}$ & 2 weeks & 0 & 0 & 1 & 0 & 1 \\
\hline in the LpMF1298 period & 3 weeks & 0 & 0 & 0 & 1 & 1 \\
\hline Total no. of subjects & & 5 & 4 & 2 & 5 & 16 \\
\hline
\end{tabular}

The difference in favor of placebo was statistically significant $(P=0.012)$.

However, the $1 \times 10^{10} \mathrm{CFU}$ dose was associated with significant formulation problems [16]. The only previous study in humans with $L$. plantarum MF1298 is a study of the survival and persistence of the strain in the gastrointestinal tract in 17 healthy volunteers. They were given $6 \times 10^{9} \mathrm{CFU}$ per day of L. plantarum MF1298 either as a freeze-dried preparation or present in $15 \mathrm{~g}$ fermented sausage. No gastrointestinal symptoms or other adverse events were spontaneously reported, but such symptoms were not systematically recorded [10]. The dose of $1 \times 10^{10} \mathrm{CFU}$ L. plantarum MF1298 selected for this study was in the same order as the doses used in other studies with lactobacilli [20]. Despite the lack of evidence for a reduced or detrimental effect of high doses, we cannot exclude that a too high dose might have contributed to the unfavourable outcome of this study.

It has been proposed that the most potent probiotics may have increased pathogenicity [21]. The multispecies probiotic preparation used in the study of acute, severe pancreatitis where mortality was increased, was composed of six strains [22]. These strains, selected from 69 different probiotic bacteria, had better probiotic properties in combination than the individual components. $L$. plantarum MF1298 had the best in vitro probiotic properties of 22 strains [8-10]. The probiotic with the best probiotic properties as determined in vitro is not

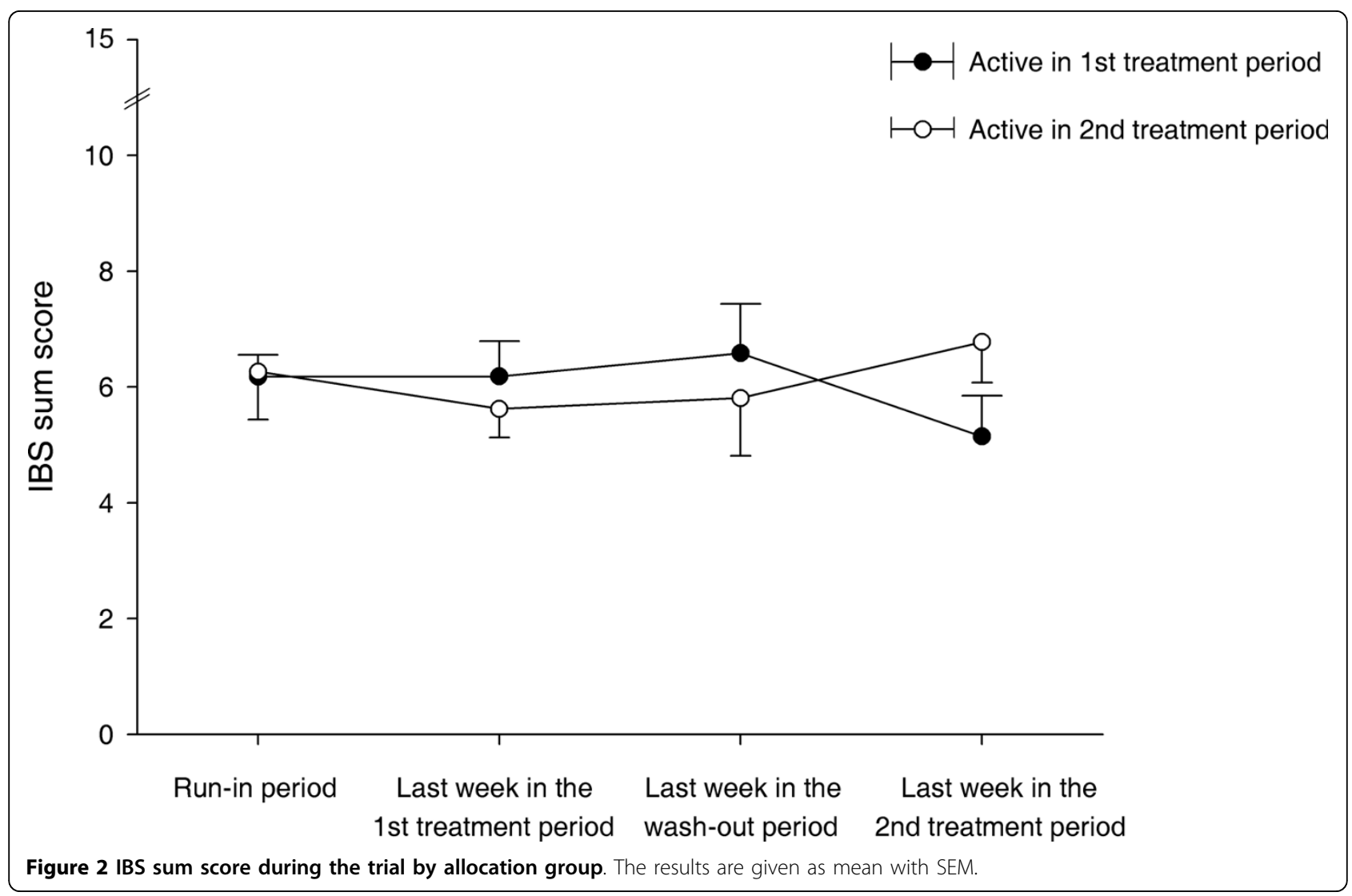


necessarily the best one to "confer a health benefit on the host".

The possibility of contamination of capsules by pathogens was excluded in our study, but the presence of endotoxins in the L. plantarum MF1298 and placebo preparations was not checked. This is, however, unlikely to be the reason for the unfavourable effect, because the company providing the capsules is a reliable producer of food supplements.

The serious adverse event and three minor adverse events reported were judged to be unrelated to the treatment.

\section{Strengths and weaknesses}

Probably due to the heterogeneity of the sample in terms of bowel habit predominance, we cannot point to aggravation of a specific symptom. But all outcomes, both the primary outcome (treatment preference) and the secondary outcomes (number of weeks with satisfactory relief of symptoms and IBS sum score), show the same unfavourable direction for active treatment. This strengthens the internal validity, but a type I error cannot be excluded.

With an IBS sum score difference of 2 chosen as clinically significant, the number needed to harm was 3.7. A 2-point difference on a scale with a range of 15 means $13 \%$, and a change of $10 \%$ is often regarded as significant on such scales. However, the IBS sum score was not validated for responsiveness and clinically significant differences. Considering that the mean IBS sum score in the run-in period was 5.97, the score difference of 2 chosen as clinically significant might be rather high.

In this study the mean age of subjects was 50 years and the proportions of subjects with diarrhoea predominant, constipation predominant, and alternating IBS were $38 \%, 6 \%$, and $56 \%$, respectively. In corresponding studies the participants were younger and the proportions of subjects in the subgroups were more balanced $[1,25]$. The older age and the somewhat different distribution of subgroups in our study raise the question of external validity. A beneficial effect in younger subjects, in subjects with more or less symptoms compared with our participants, in subgroups of subjects (such as constipation predominant), or in populations with other dietary habits and gut microflora cannot be excluded. Furthermore, a longer period of intervention would have strengthened the internal validity, but increased the drop-out rates.

The advantage of the crossover design used in this study is the increase in power of within-participant comparisons, and thus its requirement for fewer participants. For ethical reasons, the number of participants and the study period should be reduced as much as possible in a phase II study like this one. The design is fitted for stable chronic diseases [26]. Although IBS is a fluctuating disease, Figure 2 shows that the prerequisites for the use of this design were fulfilled. The IBS sum scores in the two periods were not significantly different. The detection of L. plantarum MF1298 in one faecal sample at the end of the washout period indicates that the washout period was too short in this subject. However, because the amount of L. plantarum MF1298 detected was small and the recording of symptoms took place in the last week of the three-week treatment period, the possibility for a carryover effect is negligible. In summary, the crossover design turned out to be appropriate.

\section{Conclusions}

The results from our study contribute to focus on the risks of using strains with probiotic properties without scientific evaluation. Not all strains with in vitro demonstrated probiotic properties actually "confer a health benefit on the host", and their use may even be associated with unfavourable effects. L. plantarum MF1298 might be an unfavourable strain and this should stimulate basic research on the molecular basis of probiotic properties.

\section{Acknowledgements}

We thank Dr Ole Breder, specialist in internal medicine, for his assistance in recruiting participants, and Prof Knut Rudi and research scientist Birgitte Moen at Nofima Mat AS for faecal analyses.

\section{Author details}

'Department of Medicine, Innlandet Hospital Trust, Gjøvik, Norway. ${ }^{2}$ Unit for Applied Clinical Research, Department of Cancer Research and Molecular Medicine, Norwegian University of Science and Technology, Trondheim, Norway. ${ }^{3}$ Nofima Mat AS, Ås, Norway.

\section{Authors' contributions}

SCL, LA, KN, and PGF wrote the protocol. SCL and PGF enrolled subjects and collected data and SCL imported data. SCL, SL, and PGF performed statistical analyses. SCL and PGF drafted the paper, which was reviewed by the other authors. All authors read and approved the final manuscript.

\section{Competing interests}

SCL and PGF have received funding from Nofima AS (former Matforsk AS) through $L A$ and $K N$ which in turn have received funding from Nortura BA (former Gilde BA).

Received: 20 April 2009

Accepted: 10 February 2010 Published: 10 February 2010

\section{References}

1. Spiller R, Aziz Q, Creed F, Emmanuel A, Houghton L, Hungin P, Jones R, Kumar D, Rubin G, Trudgill N, Whorwell P: Guidelines on the irritable bowel syndrome: mechanisms and practical management. Gut 2007, 56:1770-1798.

2. Chang L: Review article: epidemiology and quality of life in functional gastrointestinal disorders. Aliment Pharmacol Ther 2004, 20(Suppl 7):31-39.

3. Drossman DA: Review article: an integrated approach to the irritable bowel syndrome. Aliment Pharmacol Ther 1999, 13(Suppl 2):3-14.

4. Zoetendal EG, Vaughan EE, de Vos WM: A microbial world within us. Mol Microbiol 2006, 59:1639-1650. 
5. FAO/WHO Expert Consultation Group: Health and nutritional properties of probiotics in food including powder milk with live lactic acid bacteria Argentina: FAO/WHO 2001

6. Spiller R: Review article: probiotics and prebiotics in irritable bowel syndrome. Aliment Pharmacol Ther 2008, 28:385-396.

7. Nobaek S, Johansson ML, Molin G, Ahrne S, Jeppsson B: Alteration of intestinal microflora is associated with reduction in abdominal bloating and pain in patients with irritable bowel syndrome. Am J Gastroenterol 2000, 95:1231-1238.

8. Klingberg TD, Pedersen MH, Cencic A, Budde BB: Application of measurements of transepithelial electrical resistance of intestinal epithelial cell monolayers to evaluate probiotic activity. Appl Environ Microbiol 2005, 71:7528-7530.

9. Klingberg TD, Axelsson L, Naterstad K, Elsser D, Budde BB: Identification of potential probiotic starter cultures for Scandinavian-type fermented sausages. Int J Food Microbiol 2005, 105:419-431.

10. Klingberg TD, Budde BB: The survival and persistence in the human gastrointestinal tract of five potential probiotic lactobacilli consumed as freeze-dried cultures or as probiotic sausage. Int J Food Microbiol 2006, 109:157-159.

11. Heaton KW: Dietary fibre. BMJ 1990, 300:1479-1480.

12. Walter SD: Number needed to treat (NNT): estimation of a measure of clinical benefit. Stat Med 2001, 20:3947-3962.

13. Quigley EM: The efficacy of probiotics in IBS. J Clin Gastroenterol 2008, 42(Suppl 2):S85-\$90

14. Guyonnet D, Chassany O, Ducrotte P, Picard C, Mouret M, Mercier CH, Matuchansky C: Effect of a fermented milk containing Bifidobacterium animalis DN-173 010 on the health-related quality of life and symptoms in irritable bowel syndrome in adults in primary care: a multicentre, randomized, double-blind, controlled trial. Aliment Pharmacol Ther 2007, 26:475-486.

15. O'Mahony L, McCarthy J, Kelly P, Hurley G, Luo F, Chen K, O'Sullivan GC, Kiely B, Collins JK, Shanahan F, Quigley EM: Lactobacillus and bifidobacterium in irritable bowel syndrome: symptom responses and relationship to cytokine profiles. Gastroenterology 2005, 128:541-551.

16. Whorwell PJ, Altringer L, Morel J, Bond Y, Charbonneau D, O'Mahony L, Kiely B, Shanahan F, Quigley EMM: Efficacy of an encapsulated probiotic Bifidobacterium infantis 35624 in women with irritable bowel syndrome. Am J Gastroenterol 2006, 101:1581-1590.

17. O'Sullivan MA, O'Morain CA: Bacterial supplementation in the irritable bowel syndrome. A randomised double-blind placebo-controlled crossover study. Dig Liver Dis 2000, 32:294-301.

18. Kajander K, Myllyluoma E, Rajilic-Stojanovic M, Kyronpalo S, Rasmussen M, Jarvenpaa S, Zoetendal EG, de Vos WM, Vapaatalo H, Korpela R: Clinical trial: multispecies probiotic supplementation alleviates the symptoms of irritable bowel syndrome and stabilizes intestinal microbiota. Aliment Pharmacol Ther 2008, 27:48-57.

19. Sen S, Mullan MM, Parker TJ, Woolner JT, Tarry SA, Hunter JO: Effect of Lactobacillus plantarum $299 \mathrm{v}$ on colonic fermentation and symptoms of irritable bowel syndrome. Dig Dis Sci 2002, 47:2615-2260.

20. Montrose DC, Floch MH: Probiotics used in human studies. J Clin Gastroenterol 2005, 39:469-484

21. Boyle RJ, Robins-Browne RM, Tang MLK: Probiotic use in clinical practice: what are the risks?. Am J Clin Nutr 2006, 83:1256-1264.

22. Besselink MG, van Santvoort HC, Buskens E, Boermeester MA, van Goor $H$, Timmerman HM, Nieuwenhuijs VB, Bollen TL, van Ramshorst B, Witteman BJ, Rosman C, Ploeg RJ, Boermeester MA, Schaapherder AF, Dejong CH, Wahab PJ, van Laarhoven CJ, Harst van der E, van Eijck $\mathrm{CH}$, Cuesta MA, Akkermans LM, Gooszen HG: Probiotic prophylaxis in predicted severe acute pancreatitis: a randomised, double-blind, placebo-controlled trial. Lancet 2008, 371:651-659.

23. Niedzielin K, Kordecki H, Birkenfeld B: A controlled, double-blind, randomized study on the efficacy of Lactobacillus plantarum $299 \mathrm{~V}$ in patients with irritable bowel syndrome. Eur J Gastroenterol Hepatol 2001, 13:1143-1147.

24. Niv $E$, Naftali T, Hallak R, Vaisman N: The efficacy of Lactobacillus reuteri ATCC 55730 in the treatment of patients with irritable bowel syndromea double blind, placebo-controlled, randomized study. Clin Nutr 2005, 24:925-931.
25. Ersryd A, Posserud I, Abrahamsson H, Simren M: Subtyping the irritable bowel syndrome by predominant bowel habit: Rome II versus Rome III. Aliment Pharmacol Ther 2007, 26:953-961.

26. Woods JR, Williams JG, Tavel M: The two-period crossover design in medical research. Ann Intern Med 1989, 110:560-566.

\section{Pre-publication history}

The pre-publication history for this paper can be accessed here:http://www biomedcentral.com/1471-230X/10/16/prepub

doi:10.1186/1471-230X-10-16

Cite this article as: Ligaarden et al:: A candidate probiotic with unfavourable effects in subjects with irritable bowel syndrome: a randomised controlled trial. BMC Gastroenterology 2010 10:16.

\section{Submit your next manuscript to BioMed Central and take full advantage of:}

- Convenient online submission

- Thorough peer review

- No space constraints or color figure charges

- Immediate publication on acceptance

- Inclusion in PubMed, CAS, Scopus and Google Scholar

- Research which is freely available for redistribution

Submit your manuscript at www.biomedcentral.com/submit
C) Biomed Central 